This is a postprint version of the following published document:

Búa Núñez, Iago;Posada Román, Julio E.; Rubio-Serrano, Jesús; GarcíaSouto, José A. Instrumentation system for location of partial discharges using acoustic detection with piezoelectric transducers and optical fiber sensors. En: IEEE transactions on instrumentation and measurement, 63 (5) (May 2014), pp. 1002-1013

DOI: $\underline{10.1109 / T I M .2013 .2286891}$

(C) 2014 IEEE. Personal use of this material is permitted. Permission from IEEE must be obtained for all other uses, in any current or future media, including reprinting/republishing this material for advertising or promotional purposes, creating new collective works, for resale or redistribution to servers or lists, or reuse of any copyrighted component of this work in other works. 


\title{
Instrumentation System for Location of Partial Discharges Using Acoustic Detection With Piezoelectric Transducers and Optical Fiber Sensors
}

\author{
Iago Búa-Núñez, Julio E. Posada-Román, Jesús Rubio-Serrano, and José A. Garcia-Souto, Member, IEEE
}

\begin{abstract}
In this paper, a multichannel instrumentation system for the location of partial discharges (PDs) in power transformers is presented. It is based on the detection of the acoustic emissions from PDs in oil with several piezoelectric (PZT) and fib $r$ optic sensors. An acoustic detection and location approach is proposed based on a time reference given by one $f$ ber optic sensor installed inside the tank and the times of arrival to several PZT sensors installed outside the tank (two in front of each phase windings of a three phase transformer for a typical application). The signal processing includes digital denoising techniques and time-difference lookup table-based 3-D location algorithms; these algorithms have been implemented with virtual instrumentation. The system is tested in an acoustic experimental setup and the location accuracy is evaluated. Finally, the error propagation from the times of arrival and the influ nce of the number of sensors and their sites in the 3-D location algorithm were analyzed.
\end{abstract}

Index Terms - 3-D location algorithms, acoustic emission (AE), denoising, fib $r$ optic sensor, LabVIEW, lookup table (LUT), multichannel instrumentation system, partial discharge (PD), Peripheral Component Interconnect eXtensions for Instrumenation (PXI).

\section{INTRODUCTION}

$\mathbf{T}$ HE interest in the measurement of partial discharges (PDs) has been increased in recent years because they represent a very reliable indicator of the degradation of electrical insulation. For this reason, the monitoring of that activity is very interesting with the aim of analyzing high-voltage electrical equipment such as transformers and studying the probability of failure.

Within the methods of PD detection in transformers (electrical, Ultra High Frequency, acoustic, chemical, optical, etc.), acoustic detection has major advantages regarding electromagnetic interference immunity, it provides information for locating the source of acoustic emission (AE) and it is easy

Manuscript received June 14, 2013; revised September 19, 2013; accepted September 20, 2013. Date of publication January 9, 2014; date of current version April 3, 2014. This work was supported in part by the Spanish National Ministry of Science and Innovation under the Project DPI 2009-14628-C03-01 and in part by the scholarship FPI under Grant BES-2010-042083. The Associate Editor coordinating the review process was Dr. Edoardo Fiorucci.

The authors are with the Optoelectronics and Laser Technology Group, Department of Electronics Technology, Universidad Carlos III de Madrid, Madrid E-28911, Spain (e-mail: ibua@ing.uc3m.es; jsouto@ing.uc3m.es).

Color versions of one or more of the fgures in this paper are available online at http://ieeexplore.ieee.org.

Digital Object Identifie 10.1109/TIM.2013.2286891 to install in feld [1]-[3]. Most acoustic detection systems are focused on corona PD [4], [5]; these discharges are of higher intensity and they have a high signal to noise ratio. However, the internal PD and surface PD are less intense and their detection is greatly affected by the noise level. Fig. 1 shows the acoustic signals generated by different types of PD obtained in the laboratory.

There are several digital techniques of signal processing (digital fltering, wavelet, etc.) applied to the detection of PD, but they are focused on the electrical method of PD measurement [6]. There are scarcely a few techniques demonstrated in acoustic measurements and deployed in real applications [7]. To address the problem of internal and surface PD acoustic detection, some of these denoising techniques used in electrical PD measurements will be considered for acoustic measurements, as well as the optimal sequence of processing.

The piezoelectric (PZT) ultrasonic sensors are typically used for acoustic detection; these sensors are located externally on the walls of thetransformer tank. Currently, other techniques using sensors of optical f ber (OF) are being developed. These sensors are suitable for noisy environments with weak signals because they are embedded in the insulating medium. They can detect the acoustic signal without the inf uence of the walls acoustic interface, that causes undesirable effects like signal distortion and strong attenuation (only $10 \%$ of the acoustic signal is detected outside the tank). They are immune to electromagnetic interference. It is possible to undertake the installation of one OF sensor in each transformer in use, although it would be interesting to have a multiple installation that is possible in the fabrication process of a new transformer. The OF sensor is used for monitoring the AE of PD activity as well as it is the reference for the location process assisted by external PZT sensors. The location is done when the PD activity exceeds a threshold. The PD activity is acoustically characterized in terms of the amplitude and the number of pulses (repetition rate) and the threshold is a combination of both and their evolution with time that is determined by expert observation. Typical procedures with transformers include several PD detection methods for the assessment of the insulation; they use to compare the measurements with those obtained in laboratory tests and in the startup of the transformer onsite.

Until present different instrumentation systems and measurement methods were reported related to the detection of 

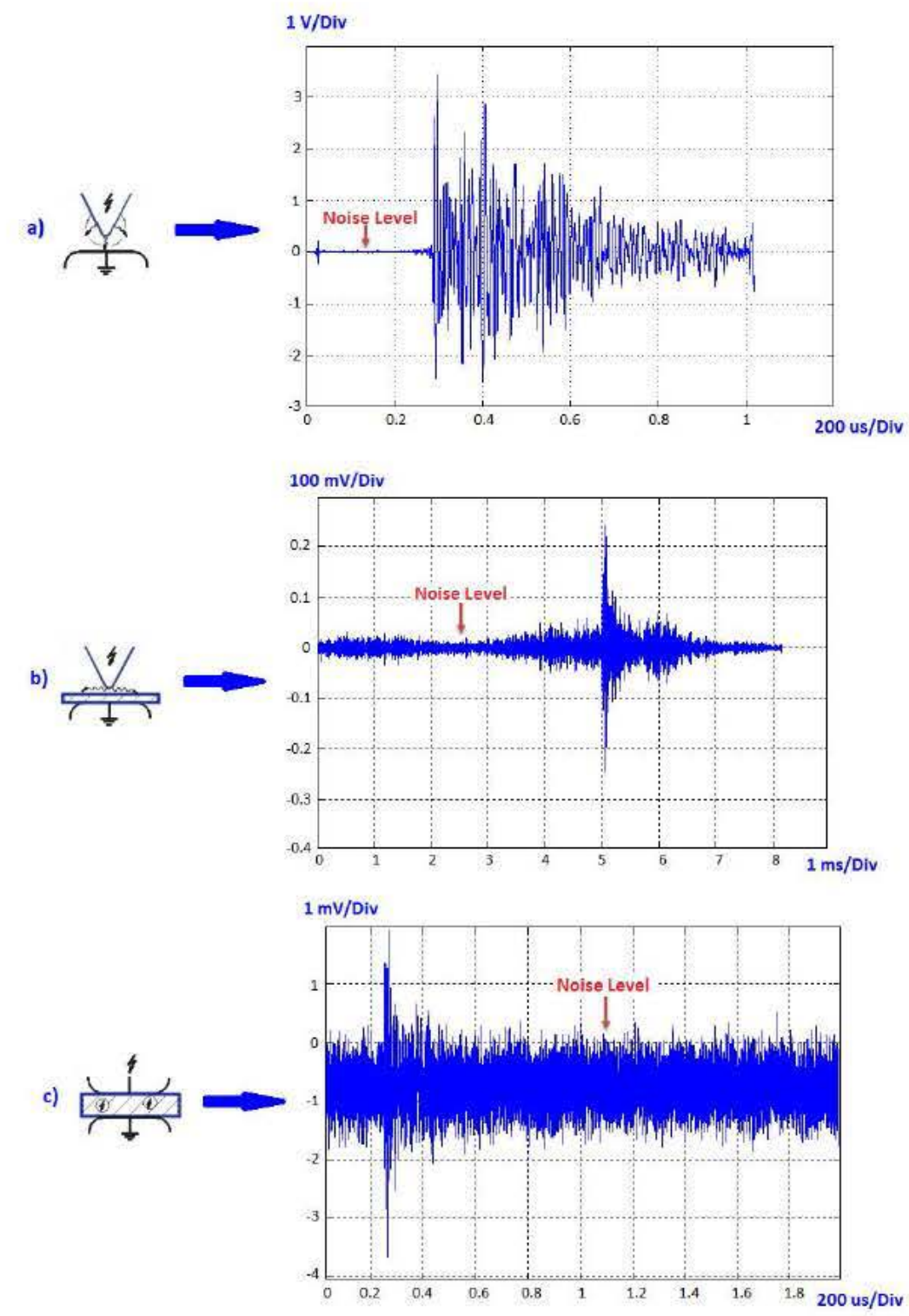

Fig. 1. Acoustic signals generated by (a) corona PD, (b) surface PD, and (c) internal PD.

PDs. They were as follows: electrical measurements and calibration of the charge [8]-[10], very high frequency [11], [12], and ultra high frequency [13]. However, the problem of defect location is still actual in power transformers because it is a complex and difficult issue. Therefore, new instrumentation systems that can improve the process of defect location and make it more reliable are important. Few results are reported on location and they are based on a highly persistent and repetitive $\mathrm{AE}$ from the damaged region [14]. This even allows auscultation of the apparatus with only one sensor [15]. However, simultaneous detection of the $\mathrm{AE}$ in several sensors and the possibility of online location is a new concept to face a predictive maintenance. In addition, AEs from sporadic PD activity and from incipient defects should be considered before a serious damage occurs.

Based on a first design of the multichannel acquisition system and denoising for the detection and location of PD using $\mathrm{AE}$, which integrates $\mathrm{OF}$ and PZT sensors and evaluate the performance of the denoising on the location problem [16]. This paper improves the data acquisition and signal processing with the aim of monitoring PD in HV three phase equipment and locating the AE source (AES). At least six PZT sensors and one OF sensor reference are integrated with a Peripheral Component Interconnect eXtensions for Instrumenation (PXI) Platform and virtual instrumentation in LabVIEW is used for this purpose.

In addition, the inclusion of a location stage in the processing is proposed. This stage integrates algorithms that allow finding out the position of the AES [17]-[21]. They are based on the time of arrival (TOA) from the $\mathrm{AE}$ to each sensor. There are different techniques for determining the TOA applied to the signals from PD, but it is common to use threshold or envelope threshold [4]. Other techniques are based on energy criterion or Akaike information criterion (AIC) [17], 


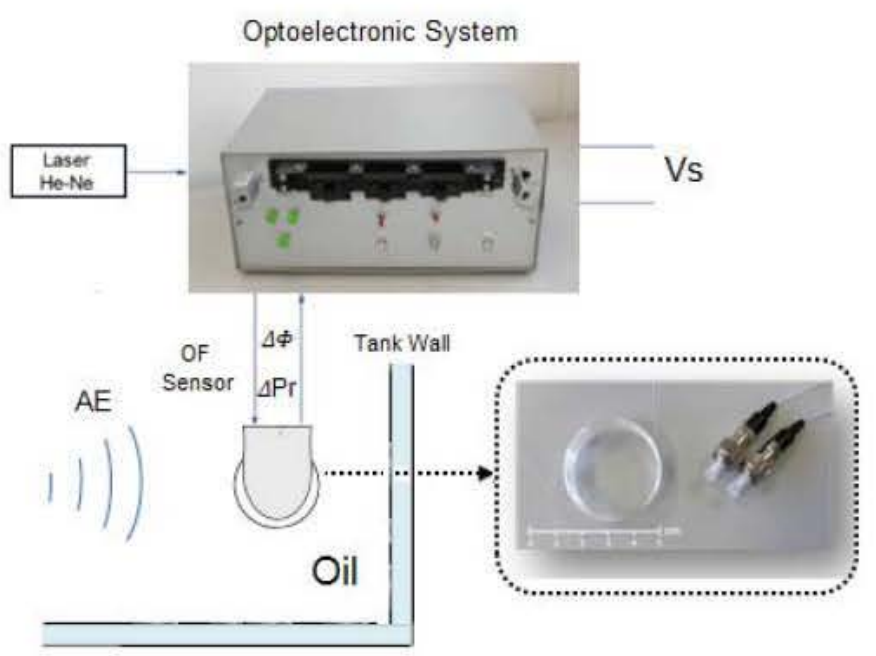

Fig. 2. Instrumentation system based on OF sensing.

universal wavelet filtering (UWF) [7], and cross correlation with a pattern waveform that can be determined from wavelet components [22]. In this paper, the algorithm is based on an automatic peak detector in which the time instant is registered when $80 \%$ of the maximum peak is reached [23].

Former work in this area undertakes global positioning system (GPS) algorithms for transformer PD source location [17]. Other studies present adaptive methods [18], iterative methods [19], and noniterative methods [20]. In this paper, the algorithm used is based on time-differences patterns. The time-differences patterns are formed for each position in the transformer by knowing the transformer internal dimensions and they are saved as lookup tables (LUTs) [21]. The source is located by comparing the measured time-differences vector with a time-difference LUT. This method always gets a single solution versus the adaptive and iterative algorithms which may not converge and versus the GPS algorithms that may obtain more than one solution. In addition, in this approach a direct mapping between the times difference of arrival and the 3-D coordinates is the only information in the LUT and therefore, the comparison with the measured ones is easier and faster. Additionally, the structure of this algorithm allows adding new sensors without additional complexity.

This paper is organized as follows. The instrumentation system used for detection and location of PD based on PZT transducers and $\mathrm{OF}$ sensors is described in Section II. The algorithm for the 3-D location of the AES is explained in Section III. The characterization of the instrumentation system with different analysis of location accuracy and error propagation is presented in Section IV. Finally, the conclusions are included in Section V.

\section{Detection of AE FRom PDs}

\section{A. Optical Fiber Sensors}

OF sensors can be embedded in the insulating medium of the transformer closer to the emission source, which allow to detect a stronger acoustic signal, and due to their wide directivity they are able to cover the entire tank. In this approach, one sensor monitors the PD activity and it generates

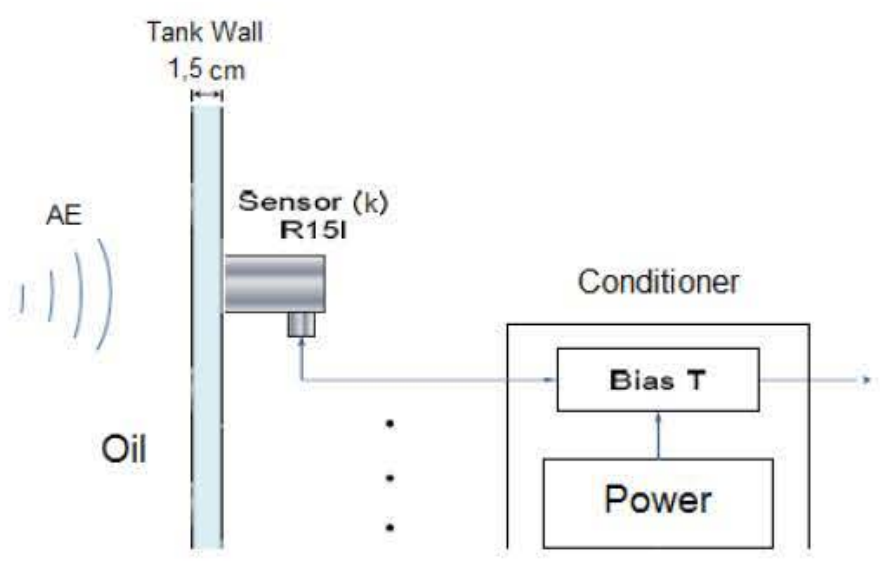

Fig. 3. Instrumentation system based on PZT sensors (R15I).

an alarm when the amplitude and the repetition rate increase above a certain level or their evolution with time get a certain slope. For example, three times the activity of the startup installation activates a warning and five times activates an alarm. It is similar in the case of considering the averaging slope. The OF sensor also provides the time reference in the location process when using external PZT acoustic sensors after a warning.

The instrumentation scheme for the OF sensor was previously designed [24] and it is shown in Fig. 2. This scheme consists of a sensor head of single-mode OF illuminated by a $\mathrm{He}-\mathrm{Ne}$ laser $(633 \mathrm{~nm})$ and an optoelectronic conditioner. It is sensitive to $\mathrm{AE}$ of $150 \mathrm{kHz}$.

The conditioning system is an interferometer, which detects the optical phase change $(\Delta \Phi)$ that is linearly proportional to the acoustic pressure change $\left(\Delta P_{r}\right)$

$$
\Delta \phi=\frac{\phi_{0}}{P_{0}} \Delta P_{r}
$$

where $P_{0}$ is a factor that depends on the strain-optic coefficients, the Young modulus and the Poisson ratio. $\Phi_{0}$ is obtained

$$
\phi_{0}=\frac{2 \pi n_{\text {eff }} L}{\lambda}
$$

where $L$ is the length of the fiber exposed to the $\mathrm{AE}, n_{\mathrm{eff}}$ is the effective refractive index of the OF, and $\lambda$ is the optical wavelength.

This device provides a voltage output $\left(V_{S}\right)$ proportional to the optical phase change $(\Delta \Phi)$ given by

$$
V_{s}=2 I_{0} \Delta \phi R \eta G_{T} G_{F}
$$

where $I_{0}$ is the mean optical power at each photodetector, $R$ is the responsivity of the photodetectors, and $\eta$ is a factor between 0 and 1 that is determined by the contrast of the interference (in this case 0.6). $G_{T}$ and $G_{F}$ are the transimpedance gain and the bandpass filter (BPF) gain, respectively.

If (1) is substituted in (3), the result is a voltage output proportional to the acoustic pressure change as follows:

$$
V_{s}=2 I_{0} \frac{\phi_{0}}{P_{0}} \Delta P_{r} R \eta G_{T} G_{F} .
$$




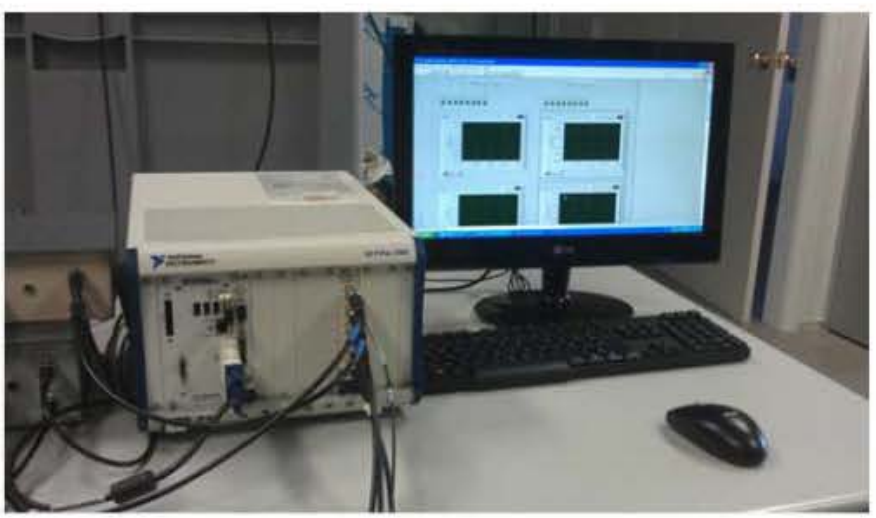

Fig. 4. Multichannel acquisition system (PXI).

\section{B. Detection With PZT Sensors}

The PZT ultrasonic sensors are typically used for acoustic detection. These sensors work with ultrasonic frequencies and are mounted externally on the walls of the transformer tank.

When the internal OF sensor detects high-PD activity the external PZT sensors help to locate the AES. However, the acoustic signal received outside the tank is much attenuated, thus the denoising is so important. The use of several sensors allows locating the source based on the TOA.

The instrumentation system based on PZT sensors is described in Fig. 3. The model of the PZT sensor is a R15I-AST (Physical Acoustic Corporation), with the following characteristics: operating range $80-200 \mathrm{kHz}$, resonant at $150 \mathrm{kHz}$, and low-noise preamplifier integrated of $40 \mathrm{~dB}$. The sensitivity of these sensors is about $1 \mathrm{~V} / \mathrm{Pa}$. Because the sensor R15I has integrated electronics but it has not separate ports for power and output, a Bias-T circuit is necessary.

\section{Multichannel Acquisition System and Denoising}

An industrial PXI has been used to integrate into the same system different types of sensors (PZT, OF, etc.) and signal processing units. The PXI system (National Instruments) is a PC-based open platform for test, measurement, and control. It includes a data acquisition module (NI PXI-5105) with eight channels of simultaneous acquisition of 12 bit resolution, $60 \mathrm{MS} / \mathrm{s}$ acquisition rate in real time, $60 \mathrm{MHz}$ bandwidth, and 128 MB onboard memory (Fig. 4). The graphical programming tool used is LabVIEW.

Different techniques from electrical PD denoising [6] were used for acoustic measurements; the most useful were wavelet techniques and digital filtering. Wavelet filtering (WT) is useful for eliminating Gaussian and white noise, improving the resolution in the detection of transient signals due to the bursts of ultrasounds as those generated by PDs. However, using only this technique, it is not possible to eliminate monochromatic and interfering noise. This is solved by a post-processing with a digital BPF in the frequency range of interest. This BPF is compliant with the IEEE standard [4] that proposes a filter with cutoff frequency of $100-300 \mathrm{kHz}$ to remove vibrations caused by pumps and fans $(<30 \mathrm{kHz})$, the Barkhausen noise $(\simeq 50 \mathrm{kHz})$, etc.

After comparing different techniques applied to acoustic detection, the most suitable system for detecting PDs is a combination in sequence of both methods (WT + BPF). This sequence is illustrated in Fig. 5.

\section{LOCATION BASED ON AE}

The common technique for 3-D spatial location of PD is the triangulation or trilateralization. This technique determines the position of the AES measuring the TOA.

Fig. 6 shows a schematic of a transformer tank with $k+1$ acoustic sensors (an internal OF sensor and $k=6$ external PZT sensors), AES (PD origin) inside and the resulting times of arrival $T$ from the OF sensor to the AES and $T_{k}$ from the $\mathrm{PZT}_{k}$ sensors to the AES.

There are several approaches to triangulation but due to all-acoustic instrumental scheme employed in this paper the time-differences approach/time difference of arrival - TDOA has been chosen.

In this approach the acoustic wave reaches the closest sensor first (assuming straight propagation) and triggers a recording process on all sensors simultaneously. Having seven sensors gives six time differences $\left(\tau_{0 \mathrm{k}}\right)$, taking as reference the first hit sensor $(T)$. In this implementation, the $\mathrm{OF}$ sensor is the time reference because it is forced to be the closest sensor to the source. Its position is selected to satisfy both of the following targets: 1) to cover the widest area that is possible and 2) to remain the closest to the source. This is feasible because of the internal installation of the OF sensor. In the case that the region is too large to be monitored by one $\mathrm{OF}$ sensor, more OF sensors could be included [25]. Fig. 7 shows the time differences of acoustic signals with unknown timing reference. The maximum duration of the time window is determined as the distance between the source and any $\mathrm{AE}$ sensor; for example, in the used tank the maximum duration would be $1 \mathrm{~ms}$ for the distance between two opposite corners.

The system of nonlinear equations of 3-D location with a time-differences approach is as follows:

$$
\begin{aligned}
& \left(x-x_{\mathrm{OF}}\right)^{2}+\left(y-y_{\mathrm{OF}}\right)^{2}+\left(z-z_{\mathrm{OF}}\right)^{2}=\left(v_{s} \cdot T\right)^{2} \\
& \left(x-x_{\mathrm{PZT} k}\right)^{2}+\left(y-y_{\mathrm{PZT} k}\right)^{2}+\left(z-z_{\mathrm{PZT} k}\right)^{2} \\
& =\left(v_{s} \cdot\left(T+\tau_{0 \mathrm{k}}\right)\right)^{2}, k=1, \ldots, 6
\end{aligned}
$$

where $v_{s}$ is the speed of sound in the medium, $x_{\mathrm{OF}}, y_{\mathrm{OF}}$, and $z_{\mathrm{OF}}$ are the coordinates of the $\mathrm{OF}$ sensor, and $x_{\mathrm{PZT} k}, y_{\mathrm{PZT} k}$, and $z_{\mathrm{PZT} k}$ are the coordinates of the $k$ th PZT sensor. The minimum number of PZT sensors must be three.

$\tau_{0 \mathrm{k}}^{\mathrm{LUT}}(x, y, z)=\frac{\left|\sqrt{\left(x-x_{\mathrm{PZT} k}\right)^{2}+\left(y-y_{\mathrm{PZT} k}\right)^{2}+\left(z-z_{\mathrm{PZT} k}\right)^{2}}-\sqrt{\left(x-x_{\mathrm{OF}}\right)^{2}+\left(y-y_{\mathrm{OF}}\right)^{2}+\left(z-z_{\mathrm{OF}}\right)^{2}}\right|}{v_{s}}, k=1, \ldots, 6$ 

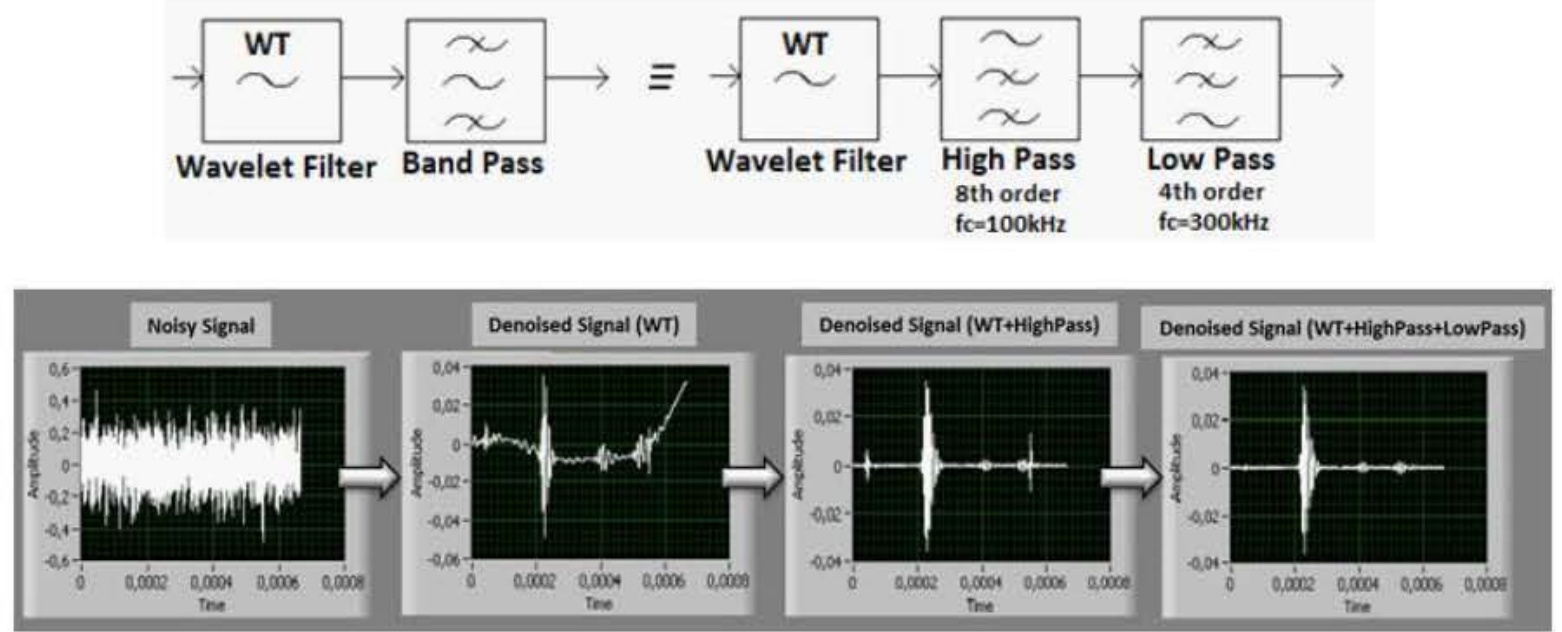

Fig. 5. Denoising sequence: wavelet and BPF. Example of operation.

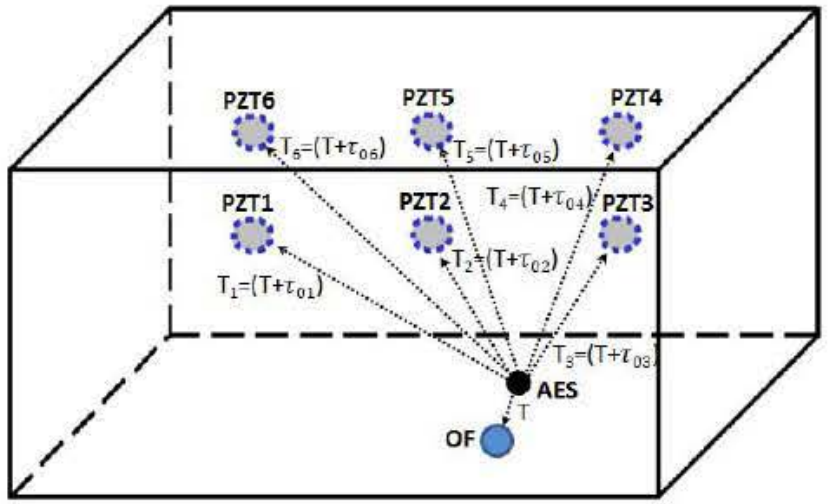

Fig. 6. Acoustic sensors on a transformer tank with the AES inside (TOA).

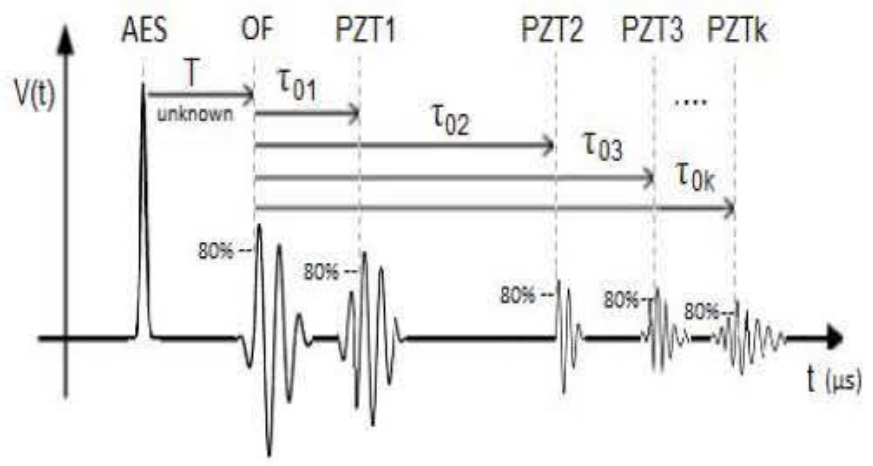

Fig. 7. Schematic of time differences with unknown time reference $T$.

The 3-D location algorithm has been implemented entirely in LabVIEW and the method is based on time-differences patterns $\left(\tau_{0 \mathrm{k}}^{\mathrm{LUT}}\right)$ [21]. The time-differences patterns are formed for each position in the transformer by knowing the transformer internal dimensions and they are saved as LUTs $(k=1, \ldots, 6$ LUTs as shown in Fig. 8). The source is located by comparing the measured time-differences vector with the time-differences LUTs.

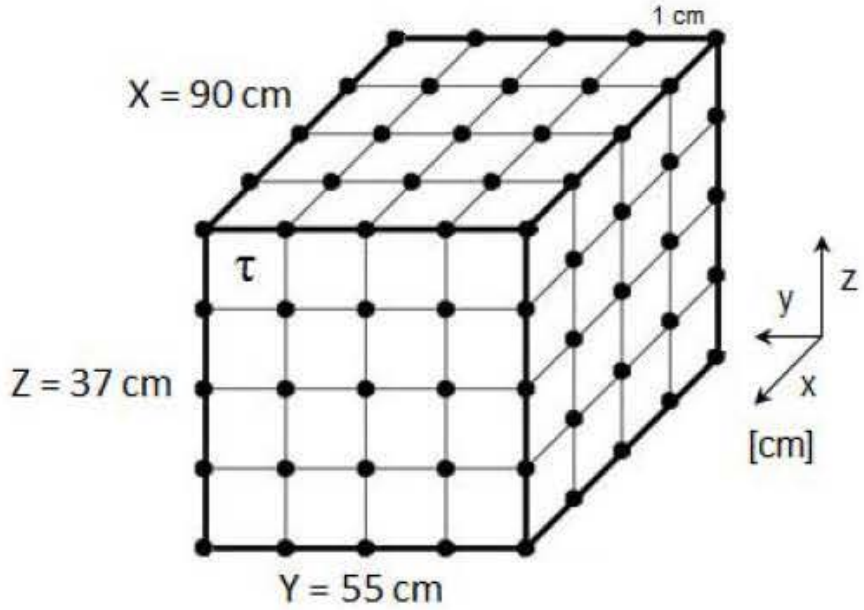

Fig. 8. Model of the transformer tank (LUT 3-D).

The time-differences LUTs are calculated by subtracting (6) and (5) and solving for the time differences $\left(\tau_{0 \mathrm{k}}\right)$, as shown in (7) at the bottom of the previous page.

The most suitable solution is one that fulfills

$$
\begin{aligned}
& (x, y, z) / \min \left(\sum_{k=1}^{6}\left|\tau_{0 \mathrm{k}}^{\mathrm{LUT}}(x, y, z)-\tau_{0 \mathrm{k}}\right|\right), \\
& x \in[0,90) \quad y \in[0,55) \quad z \in[0,37)
\end{aligned}
$$

where $(x, y, z)$ is the AES site, $\tau_{0 \mathrm{k}}$ are the measured time differences, $\tau_{0 \mathrm{k}}^{\mathrm{LUT}}(x, y, z)$ are the time-differences LUTs.

The flowchart of the location process is explained in Fig. 9 and it consists of four stages. A first stage of initialization, the LUTs 3-D [ $\left.\tau_{0 \mathrm{k}}^{\mathrm{LUT}}(x, y, z)\right]$ are built through (7) considering the speed of sound in the medium, the tank dimensions and the coordinates of the sensors. The second stage of measurement and reading, on the one hand the LUTs $3-\mathrm{D}\left[\tau_{0 \mathrm{k}}^{\mathrm{LUT}}(x, y, z)\right]$ are read and on the other hand the time differences $\left(\tau_{0 \mathrm{k}}\right)$ are measured. A third stage of operation and searching, first the time deviation is calculated by subtracting the times measured to the 


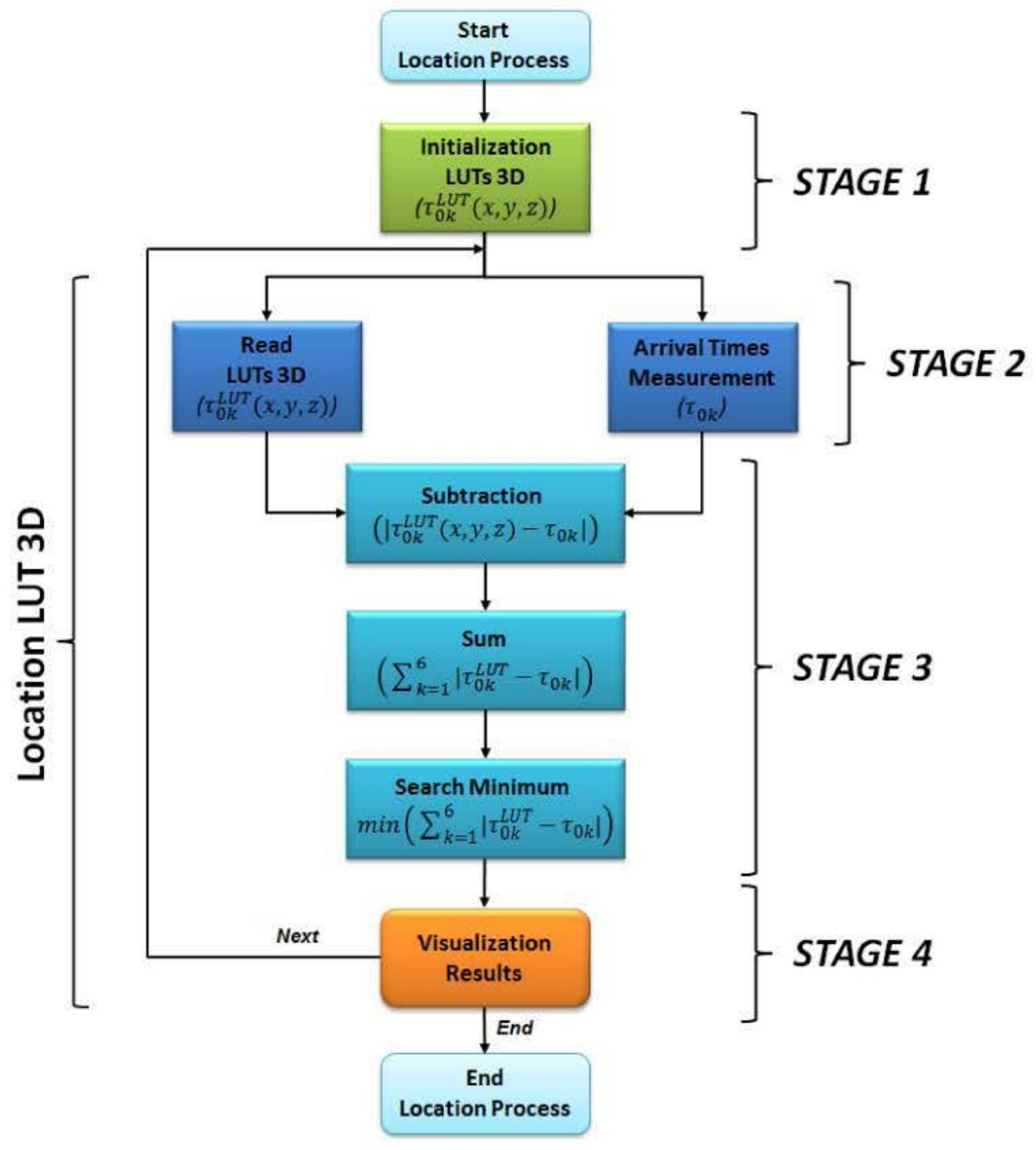

Fig. 9. Flowchart for the location process.

time-differences LUTs for each PZT sensor $\left(\mid \tau_{0 \mathrm{k}}^{\mathrm{LUT}}(x, y, z)-\right.$ $\tau_{0 \mathrm{k}} \mid$ ), second these matrices (tables) of time deviation are added $\left(\sum_{k=1}^{6}\left|\tau_{0 \mathrm{k}}^{\mathrm{LUT}}(x, y, z)-\tau_{0 \mathrm{k}}\right|\right)$ and finally the minimum time deviation is searched in the sum matrix (8). In the last stage the results of the location process are displayed and stored.

Two alternatives are also proposed in Fig. 10. The first one uses search by sections, that is to say the tank is divided in sections associated with each PZT sensor (Fig. 11), and the solution is only searched in the closest PZT section [min $\left(\tau_{0 \mathrm{k}}\right)$ ]. This method reduces the time of searching and it is directly connected with the real application in a three phase transformers. In a system with six sensors (six sections), the time of searching would be reduced in a factor 6 .

The second alternative is divided into two parts of progressive refinement. A first part performs a coarse 3-D location in $\mathrm{dm}(9 \times 6 \times 4 \mathrm{dm})$ and a second part performs a finer 3 -D location in $\mathrm{cm}(1 \times 1 \times 1 \mathrm{dm}=10 \times 10 \times 10 \mathrm{~cm})$ within the solution of the first part. This method reduces the computation time. In this case, the dimensions of the LUTs will be $(9 \times 6 \times 4)+(10 \times 10 \times 10)=$ 1216 versus $90 \times 55 \times 37=183150$ of the standard method.

\section{Characterization OF THE INSTRUMENTATION SYSTEM}

\section{A. Experimental Setup}

With the aim of producing a real acoustic environment that emulates the $\mathrm{AE}$ from PDs in a transformer, an experimental platform has been built. It consists mainly of a tank with a cubic shape, with dimensions of $103 \mathrm{~cm} \times$ $63 \mathrm{~cm} \times 63 \mathrm{~cm}$. The walls of the tank are made of polymethylmethacrylate (PMMA) and have a thickness of $1.5 \mathrm{~cm}$. One advantage of using this material is that it allows greater coupling between the acoustic signal and the external sensors. To avoid reflections which interfere with the correct measurement of some parameters of the experiment, an acoustic 


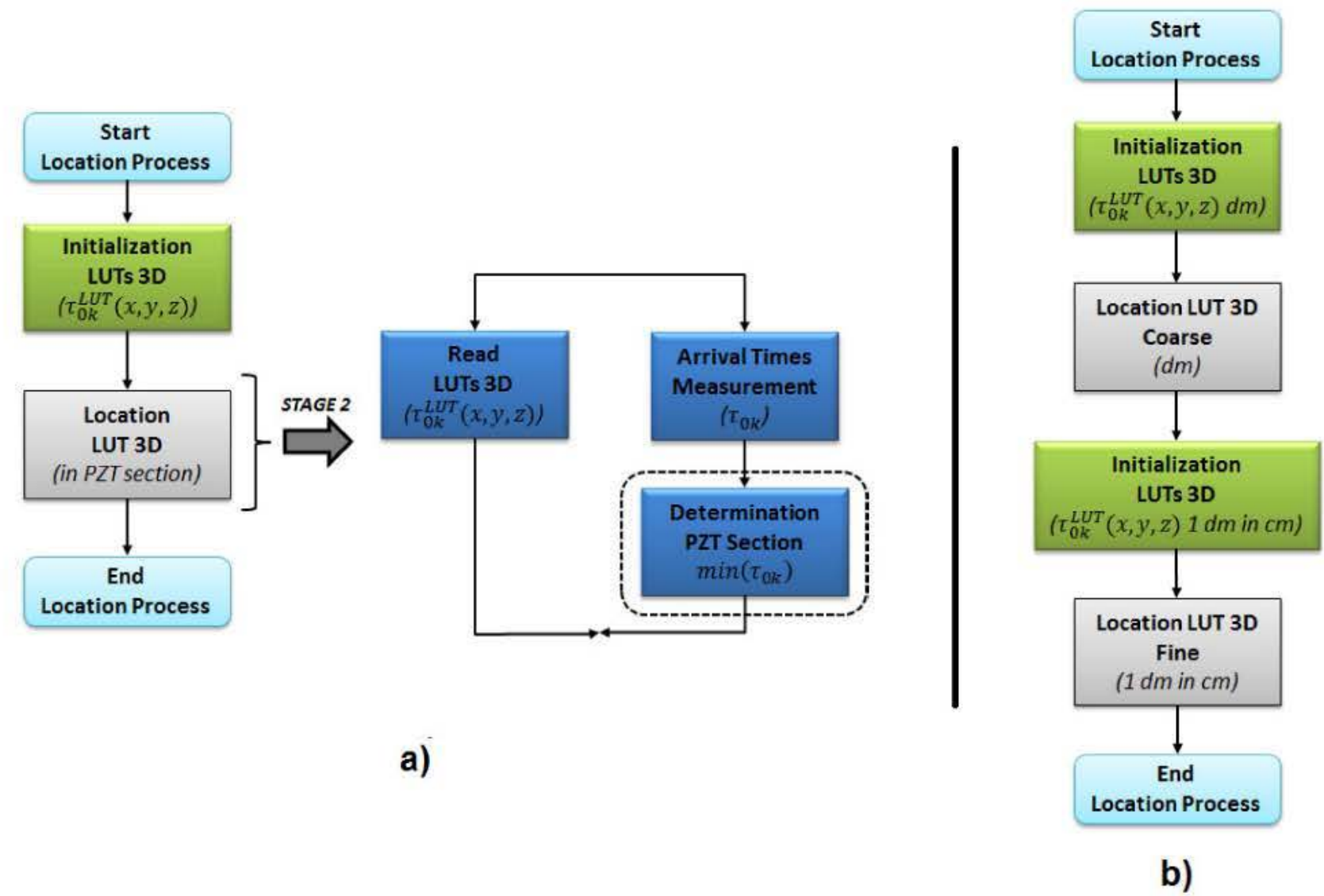

Fig. 10. Flowcharts of two proposed alternatives for the location process. (a) Search by sections. (b) Search by progressive refinement.

absorbing material of polyurethane rubber (Apltile SF5048 of Precision Acoustics-U.K.) with $5 \mathrm{~cm}$ thick fixed to the inner face of some of the tank walls has been used. The tank is filled with water up to $37 \mathrm{~cm}$. As a result, the internal dimensions are $90 \mathrm{~cm} \times 55 \mathrm{~cm} \times 37 \mathrm{~cm}$, like the model in Fig. 8.

The PMMA is optimized for the acoustic testing and the characterization of the instrumentation system. However, some differences are anticipated when comparing with a real transformer metallic tank: 1) the attenuation of the acoustic signal coming through the wall and 2) the road to the sensor of the acoustic signal propagating inside the wall. Regarding the attenuation, the OF sensor is not affected because it is located inside. In addition, the PMMA reduces the acoustic impedance mismatch with the internal medium and therefore, the attenuation in the PMMA is much lower. To account for the additional attenuation in real conditions, tests of detection at the limit of three times the amplitude of the noise have been made. If the signal is three times the amplitude of the noise or larger, the dispersion of the TOA to each PZT is less than $1 \%$ in all sensors. If the signal is even lower but distinguishable from noise after processing, the dispersion of the TOA is less than $10 \%$. This has been experimentally evaluated. In regard to the different paths to the sensor, again the OF sensor is not affected because it is immersed. In the case of the PZT sensors, the PMMA eliminates the alternative path through the walls because the sound propagation velocity (transversal) for PMMA $(1378 \mathrm{~m} / \mathrm{s})$ is lower than the sound propagation velocity for oil $(1410 \mathrm{~m} / \mathrm{s})$. This is different in a metallic tank, but in that case the filtering of low frequencies is able to attenuate the observation of paths through the walls. Additional work is to analyze the effect of multiple paths [2].

To emulate the PDs a wave generator applied to a PZT ultrasonic transducer hydrophone (B\&K 8103) working as emitter has been employed. The characteristics of the ultrasonic signals that are generated by the hydrophone are similar to those of the AEs from PDs [22].

In this case, six PZT ultrasonic sensors (R15I) fixed to the outer face of one wall of the tank and an OF sensor immersed in the insulating medium have been used. The minimum number of sensors must be three PZT sensors outside and the OF sensor inside. However, a set of two sensors per phase (six sensors) and the OF sensor is chosen considering a three phase scheme (Fig. 11). Fig. 12 shows the experimental scheme implemented. It is composed mainly by the tank instrumented with PZT sensors on the walls, the OF sensor inside and a multichannel measurement system. The latter has hardware of conditioning and acoustic signal acquisition, and software that provides the denoising of the acoustic signals and the location of the AES. The positions of the sensors are shown in Table I.

Fig. 13 shows the experimental platform for acoustic measurements. To analyze the performance of the instrumentation 

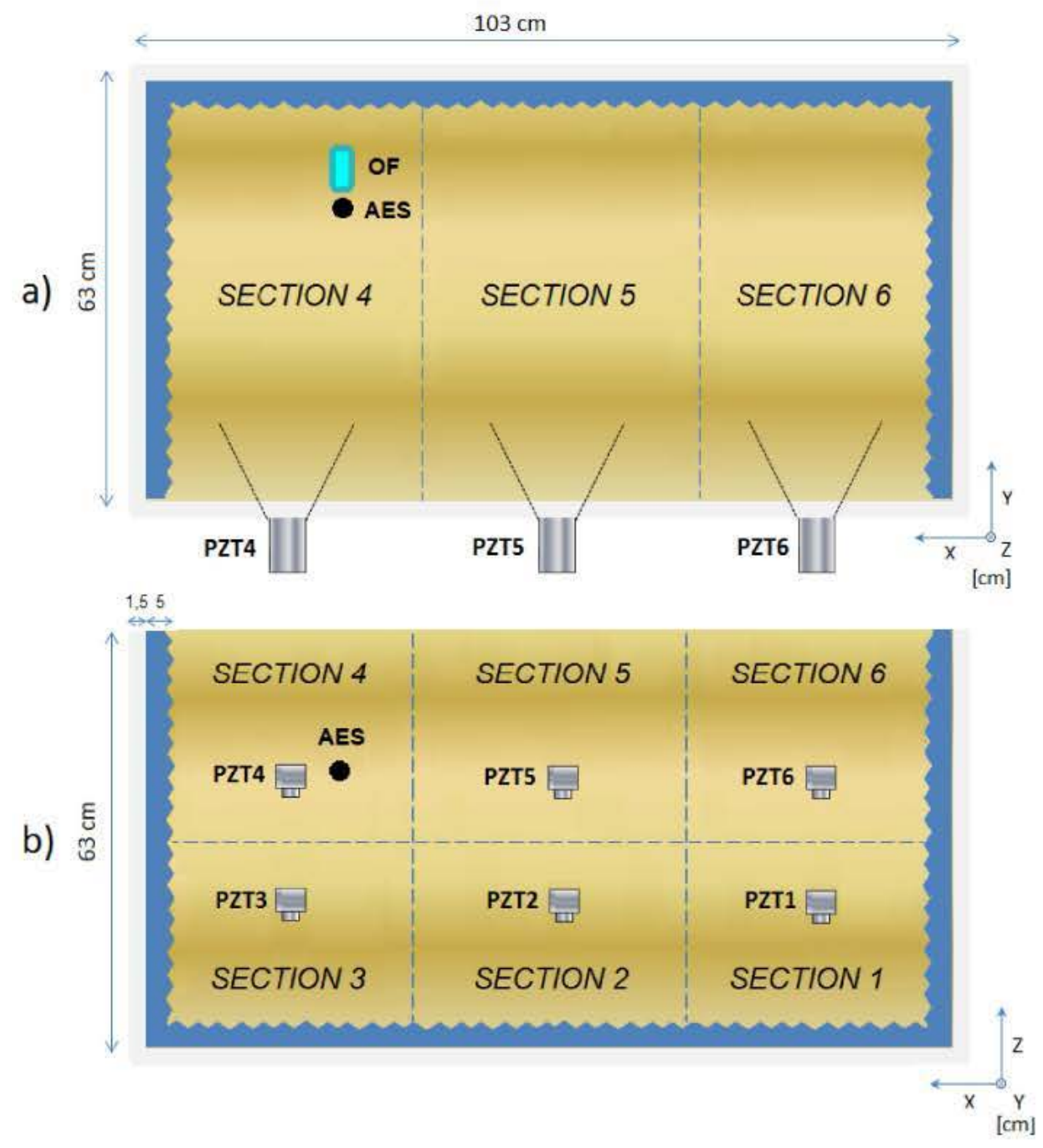

Fig. 11. Experimental platform for the location of the emission source. (a) Top view. (b) Front view.

system to detect simultaneously the $\mathrm{AE}$ in all sensors and locate the AES, two types of experiments were carried out on this platform, which are described in the following sections.

\section{B. Analysis of the Location Accuracy}

The objective is to test a schematic of multiple sensors to analyze the location accuracy of AEs. With that aim the 3-D location algorithm, described in Fig. 9, is applied to the measured TOA of 41 AEs to each and every sensor. The installation (as it is shown in Figs. 12 and 13) was used to carry out this experiment, using an OF sensor immersed in the tank and six PZT sensors installed on the walls.

The emitter was placed at $\left(X_{\mathrm{AES}}, Y_{\mathrm{AES}}, Z_{\mathrm{AES}}\right)$ and a voltage burst $(150 \mathrm{kHz}$ and $6 \mathrm{Vpk}$-pk) was applied to it. The 3-D location algorithm was applied to the TOA from the 41 acoustic measures that are obtained for each sensor.

Fig. 14 shows the 3-D representation of the resolved position of the AES that is displayed in the instrumentation system. The circles represent solutions from minimum number of events in green to maximum number of events in blue, the orange-cubes represent the sensors and the red-x represents the real position of the AES (calibrated position). As can be seen, 39 of 41 solutions resolve the same point for the location of the AES with a resolution of $1 \mathrm{~cm}$. In addition, these 39 solutions match the calibrated position $\left(X_{\mathrm{AES}}, Y_{\mathrm{AES}}\right.$, $\left.Z_{\mathrm{AES}}\right)=(66,43,26)$.

For a better understanding of the execution of the 3-D location algorithm, a 2-D representation $(X Z)$ of the matrix of time deviation $\left[M(x, y=43, z)=\sum_{k=1}^{6} \mid \tau_{0 \mathrm{k}}^{\mathrm{LUT}}(x, y=\right.$ $\left.\left.43, z)-\tau_{0 \mathrm{k}} \mid\right)\right]$ was done by fixing the coordinate $y$ to the real value of the AES $(y=43)$. It is shown in Fig. 15, where the representation of the gradient to the solution is done as follows:

$$
M^{\prime}(x, y=43, z)=\frac{\max (M(x, y=43, z))}{M(x, y=43, z)} .
$$

In Fig. $15 x$-axis is the $x$-coordinate, $y$-axis is the $z$-coordinate and $z$-axis is the value of the matrix. It shows how the values grow when they are closer to the solution (maximum). 
EXPERIMENTAL PLATFORM

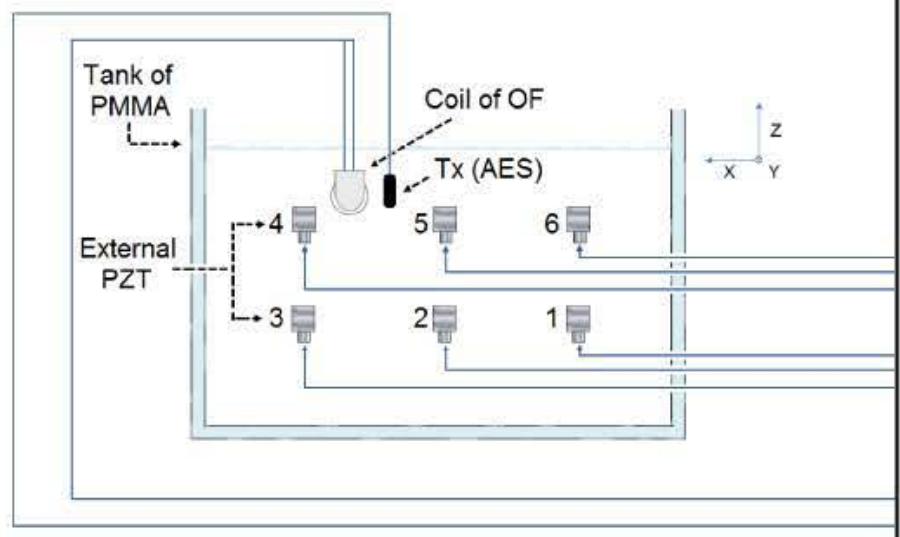

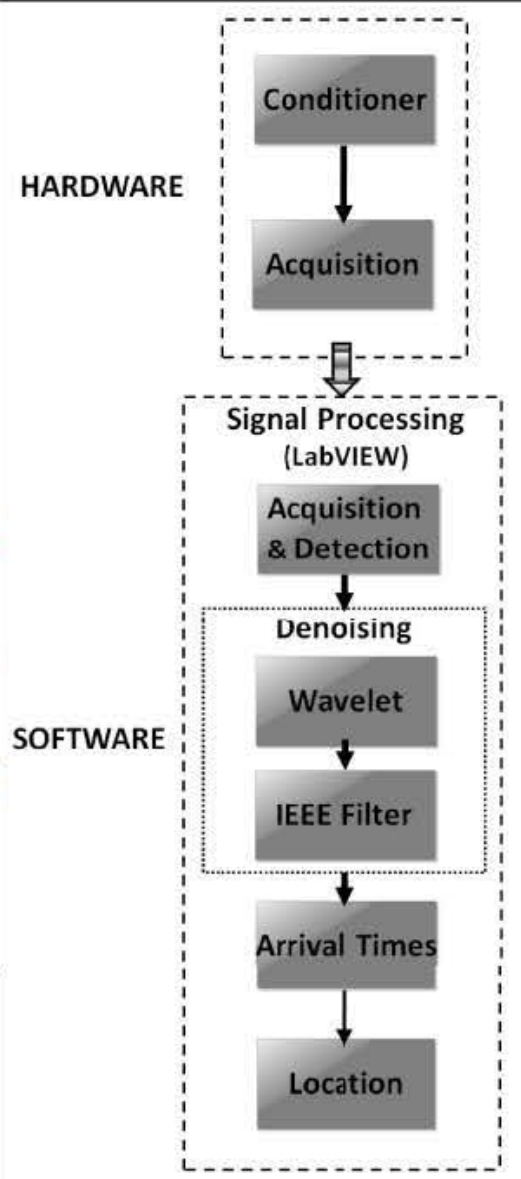

L.

Fig. 12. Multichannel instrumentation system for the detection and location of PDs.

TABLE I

POSITION OF THE SENSORS

\begin{tabular}{|c|c|c|c|c|c|c|c|}
\hline Sensor & OF & PZT1 & PZT2 & PZT3 & PZT4 & PZT5 & PZT6 \\
\hline $\begin{array}{c}\text { Position } \\
\text { x,y,z }(\mathrm{cm})\end{array}$ & $66,50,26$ & $22,0,13$ & $44,0,13$ & $67,0,13$ & $67,0,24$ & $44,0,24$ & $22,0,24$ \\
\hline
\end{tabular}

\section{Error Propagation From the Times of Arrival to the Location Algorithm}

The algorithm sensitivity to errors in the TOA was evaluated. A delay of $1 \%$ of the full scale $(3 \mu \mathrm{s})$ and $10 \%$ of the full scale $(30 \mu \mathrm{s})$ is introduced in the TOA. The limiting error is calculated as follows:

$$
\begin{aligned}
\text { Error }= & \left|E_{x}\right|+\left|E_{y}\right|+\left|E_{z}\right|=\left|X_{\mathrm{AES}}-X_{\mathrm{SOL}}\right| \\
& +\left|Y_{\mathrm{AES}}-Y_{\mathrm{SOL}}\right|+\left|Z_{\mathrm{AES}}-Z_{\mathrm{SOL}}\right|, \quad[\mathrm{cm}]
\end{aligned}
$$

where $X_{\mathrm{AES}}, Y_{\mathrm{AES}}$, and $Z_{\mathrm{AES}}$ are the coordinates of the real position of the AES and $X_{\mathrm{SOL}}, Y_{\mathrm{SOL}}$, and $Z_{\mathrm{SOL}}$ are the coordinates of the solution of the algorithm.

Fig. 16 is a representation of the maximum limiting error for a delay of $1 \%$ and $10 \%$ in one or more sensors. When the delay of $1 \%$ or $10 \%$ is introduced in the TOA of each sensor except the closest ones to the AES, the limiting error was less

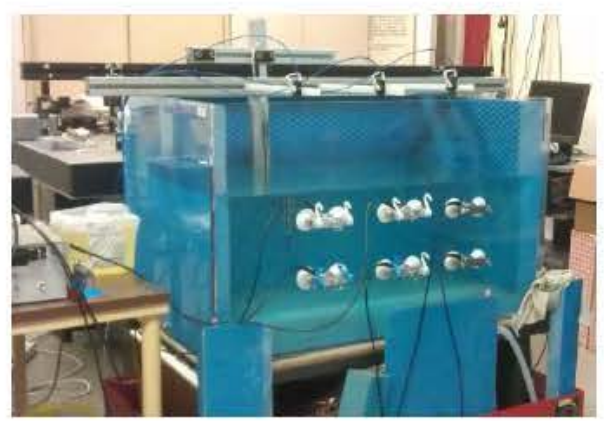

Fig. 13. Experimental platform for acoustic tests.

than $1 \mathrm{~cm}$. When the sensors affected by the delay are the closest ones to AES, the limiting error increases in function of the number of sensors involved in the analyzed cause that the delay is $10 \%$. In the particular case of a systematic error in all sensors (time zero deviation), the influence in the solution of the algorithm is smaller.

\section{Influence of the Number of Sensors and Their Sites}

The sensitivity of location algorithm to the number of sensors and their sites was evaluated. For that purpose, the 


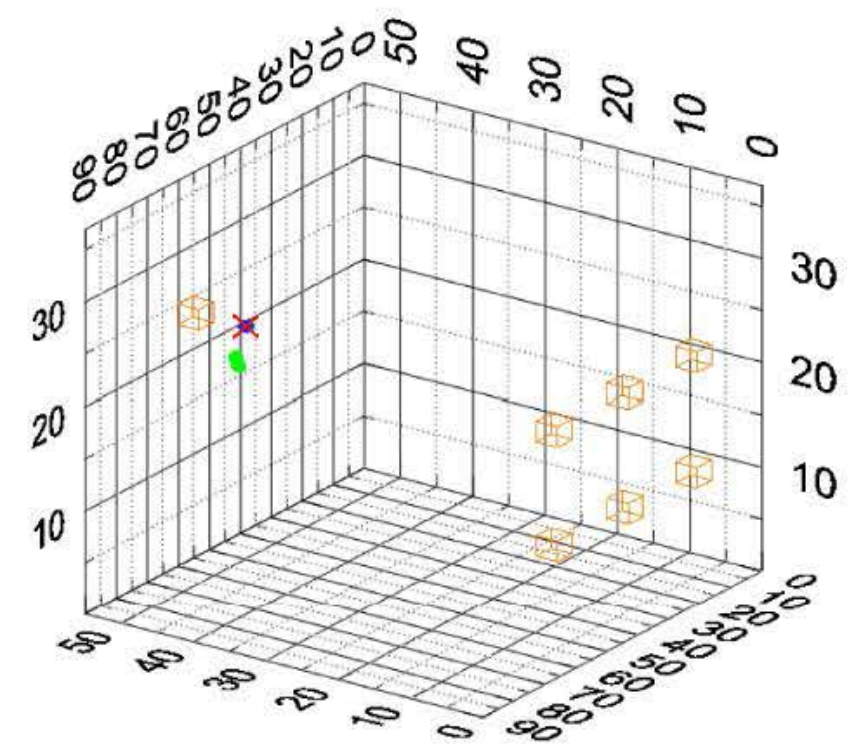

Fig. 14. Location of the emission source.

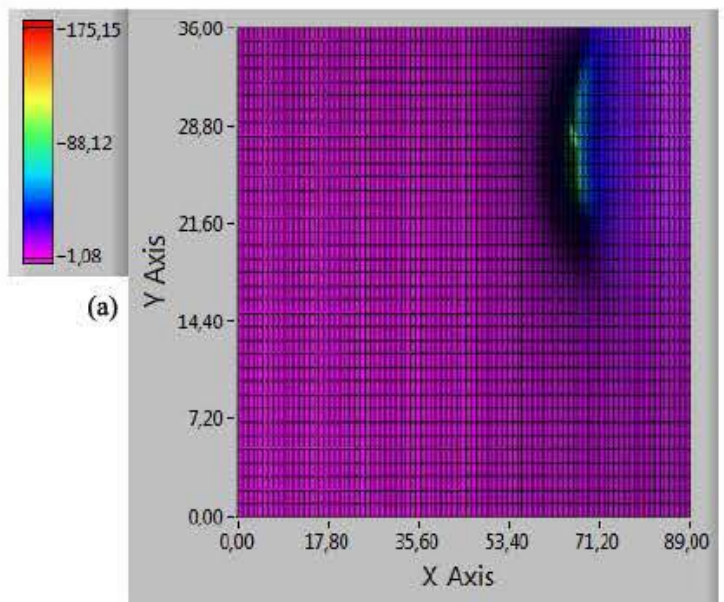

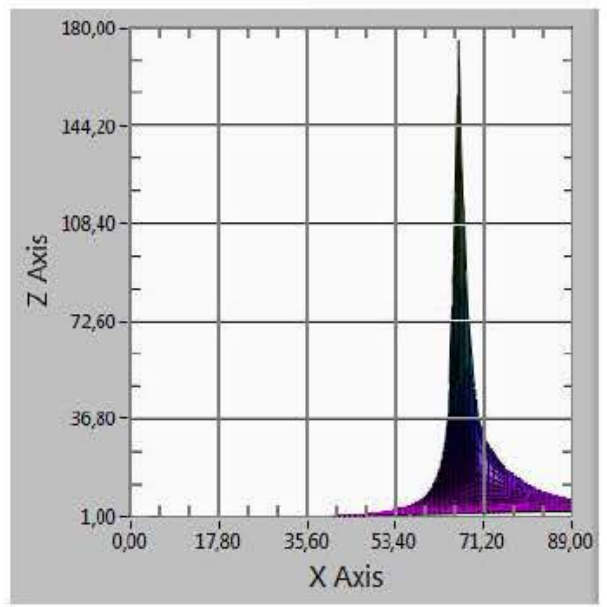

(b)

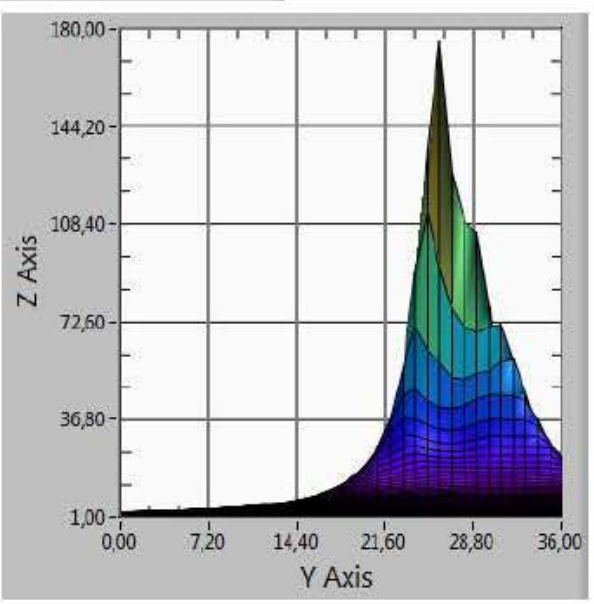

(c)

Fig. 15. Location of AE based on time deviation matrix. Representation of the gradient to the solution. (a) 2-D plot at constant value of $y$ coordinate. (b) $\mathrm{XZ}$ projection. (c) $Y Z$ projection.

answer of the algorithm is analyzed when one or more sensors are removed.

Fig. 17 is a representation of the maximum limiting error when one or more sensors are removed. If the closest sensors to the AES are not removed the result of the location algorithm is not affected. If the closest sensors to the source are removed, the maximum limiting error is the represented one in the figure. In all cases the limiting error does not exceed $7 \mathrm{~cm}$. 


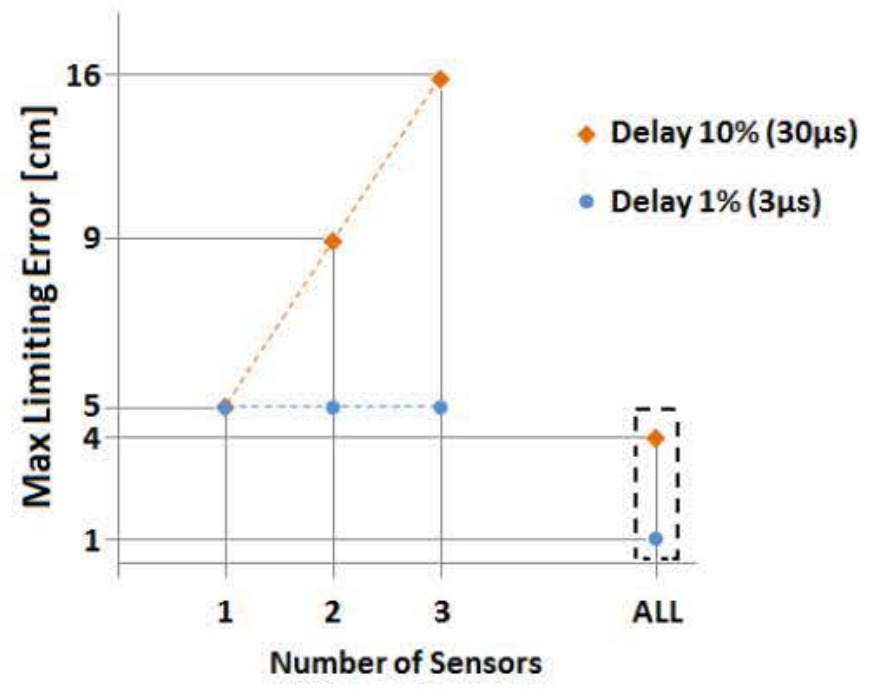

Fig. 16. Representation of the maximum limiting error for a delay of $1 \%$ and $10 \%$ in one or more sensors.

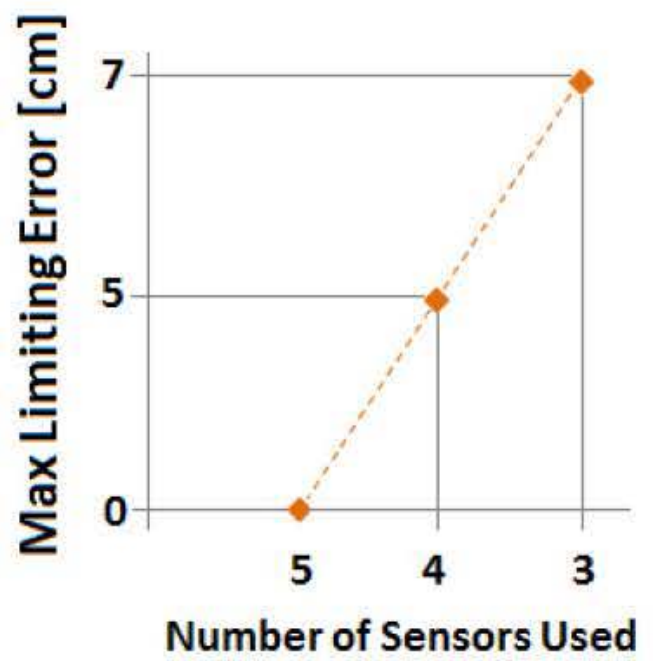

Fig. 17. Representation of the maximum limiting error when one or more sensors are removed.

\section{CONCLUSION}

In this paper, a multichannel instrumentation system for the location of PDs in power transformers has been presented. It is based on the detection of the AEs from PDs in oil with several PZT and fiber optic sensors. An all-acoustic detection and location approach has been proposed based on a time reference given by one fiber optic sensor installed inside the tank and the times of arrival to several PZT sensors installed outside the tank (two in front of each phase windings of a three phase transformer for a typical application). The signal processing includes digital denoising techniques and timedifference LUT-based location algorithms implemented with virtual instrumentation. In short, a complete tool that detects and locates AEs generated by PDs has been designed and it is accurate to $1 \mathrm{~cm}$.

Additionally, an analysis of the error propagation from the times of arrival is done, as well as the influence of the number of sensors and their sites in the 3-D location algorithm. This analysis shows that the limiting error is less than $1 \mathrm{~cm}$ when the sensors that are closest to the AES are not removed.

\section{REFERENCES}

[1] L. E. Lundgaard, "Partial discharge. XIII. Acoustic partial discharge detection-fundamental considerations," IEEE Electr. Insul. Mag., vol. 8 , no. 4, pp. 25-31, Jul./Aug. 1992.

[2] L. E. Lundgaard, "Partial discharge. XIV. Acoustic partial discharge detection-practical application," IEEE Electr. Insul. Mag., vol. 8, no. 5, pp. $34-43$, Sep./Oct. 1992.

[3] P. M. Eleftherion, "Partial discharge. XXI. Acoustic emission-based PD source location in transformers," IEEE Electr. Insul. Mag., vol. 11, no. 6, pp. 22-26, Nov./Dec. 1995.

[4] IEEE Guide for the Detection and Location of Acoustic Emissions from Partial Discharges in Oil-Immersed Power Transformers and Reactors, IEEE Standard C57.127, 2007.

[5] R. Bartnikas, "Detection of partial discharges (Corona) in electrical apparatus," IEEE Trans. Electr. Insul., vol. 25, no. 1, pp. 111-124, Feb. 1990.

[6] S. Sriram, S. Nitin, K. M. M. Prabhu, and M. J. Bastiaans, "Signal denoising techniques for partial discharge measurements," IEEE Trans. Dielectr. Electr. Insul., vol. 12, no. 6, pp. 1182-1191, Dec. 2005.

[7] T. Boczar and D. Zmarzly, "Application of wavelet analysis to acoustic emission pulses generated by partial discharges," IEEE Trans. Dielectr. Electr. Insul., vol. 11, no. 3, pp. 433-449, Jun. 2004.

[8] S. N. Hettiwatte, Z. D. Wang, and P. A. Crossley, "Investigation of propagation of partial discharges in power transformers and techniques for locating the discharge," IEE Proc. Sci. Meas. Technol., vol. 152, no. 1, pp. 25-30, Jan. 2005.

[9] T. K. Abdel-Galil, R. M. Sharkawy, M. M. A. Salama, and R. Bartnikas, "Partial discharge pattern classification using the fuzzy decision tree approach," IEEE Trans. Instrum. Meas., vol. 54, no. 6, pp. 2258-2263, Dec. 2005.

[10] M. Chiampi, G. Crotti, Y. Hu, and A. Sardi, "Development and application of a programmable partial discharge calibrator," IEEE Trans. Instrum. Meas., vol. 58, no. 4, pp. 768-774, Apr. 2009.

[11] G. Robles, J. M. Martínez-Tarifa, M. V. Rojas-Moreno, and J. Sanz-Feito, "Inductive sensor for measuring high frequency partial discharges within electrical insulation," IEEE Trans. Instrum. Meas., vol. 58, no. 11, pp. 3907-3913, Nov. 2009.

[12] M. V. Rojas-Moreno, G. Robles, B. Tellini, C. Zappacosta, J. M. Martínez-Tarifa, and J. Sanz-Feito, "Study of an inductive sensor for measuring high frequency current pulses," IEEE Trans. Instrum. Meas., vol. 60, no. 5, pp. 1893-1900, May 2011.

[13] G. Robles, M. Sánchez-Fernández, R. A. Sánchez, M. V. Rojas-Moreno, E. Rajo-Iglesias, and J. M. Martínez-Tarifa, "Antenna parametrization for the detection of partial discharges," IEEE Trans. Instrum. Meas., vol. 62, no. 5, pp. 932-941, May 2013.

[14] R. A. Hooshmand, M. Parastegari, and M. Yazdanpanah, "Simultaneous location of two partial discharge sources in power transformers based on acoustic emission using the modified binary partial swarm optimisation algorithm," IET Sci. Meas. Technol., vol. 7, no. 2, pp. 119-127, Mar. 2013.

[15] W. Sikorski, K. Siodl, H. Moranda, and W. Ziomek, "Location of partial discharge sources in power transformers based on advanced auscultatory technique," IEEE Trans. Dielectr. Electr. Insul., vol. 19, no. 6, pp. 1948-1956, Dec. 2012.

[16] I. Búa-Núnez, J. E. Posada-Román, J. Rubio-Serrano, and J. A. García-Souto, "Multichannel acquisition system and denoising for the detection and location of partial discharges using acoustic emissions," in Proc. IEEE 12MTC13, May 2013, pp. 1135-1140.

[17] S. M. Markalous, S. Tenbohlen, and K. Feser, "Detection and location of partial discharges in power transformers using acoustic and electromagnetic signals," IEEE Trans. Dielectr. Electr. Insul., vol. 15, no. 6, pp. 1576-1583, Dec. 2008.

[18] G. Vakulya and G. Simon, "Fast adaptive acoustic localization for sensor networks," IEEE Trans. Instrum. Meas., vol. 60, no. 5, pp. 1820-1829, May 2011.

[19] J. Ramírez-Niño and A. Pascacio, "Acoustic measuring of partial discharge in power transformers," Meas. Sci. Technol., vol. 20, no. 11, pp. 115108-1-115108-3, Nov. 2009. 
[20] P. Kundu, N. K. Kishore, and A. K. Sinha, "A non-iterative partial discharge source location method for transformers employing acoustic emission techniques," Appl. Acoust., vol. 70, nos. 11-12, pp. 1378-1383, Dec. 2009.

[21] Y. Lu, X. Tan, and X. Hu, "PD detection and localization by acoustic measurements in an oil-filled transformer," IEE Proc. Sci. Meas. Technol., vol. 147, no. 2, pp. 81-85, Mar. 2000.

[22] J. Rubio-Serrano, M. V. Rojas-Moreno, J. Posada, J. M. Martínez-Tarifa, G. Robles, and J. A. García-Souto, "Electro-acoustic detection, identification and location of partial discharge sources in oil-paper insulation systems," IEEE Trans. Dielectr. Electr. Insul., vol. 19, no. 5, pp. 1569-1578, Oct. 2012.

[23] J. Rubio-Serrano, J. Posada, I. Búa, and J. A. García-Souto, "Comparison of processing techniques for optimizing the diagnosis of solid insulation based on acoustic emissions from partial discharges," in Proc. IEEE ICSD, Jun. 2013, pp. 129-132.

[24] J. Posada-Roman, J. A. Garcia-Souto, and J. Rubio-Serrano, "Fiber optic sensor for acoustic detection of partial discharges in oil-paper insulated electrical systems," Sensors, vol. 12, no. 4, pp. 4793-4802, Apr. 2012.

[25] J. E. Posada, J. A. Garcia-Souto, and J. Rubio-Serrano, "Multichannel optical-fibre heterodyne interferometer for ultrasound detection of partial discharges in power transformers," Meas. Sci. Technol., vol. 24, no. 9, pp. 1-9, Jul. 2013.

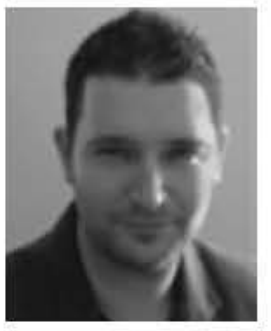

Iago Búa-Núñez was born in Pontevedra, Spain, in 1982. He received M.Sc. degree in telecommunications engineering from Universidad de Vigo, Pontevedra, in 2009, and the M.Sc. degree in electric, electronic and automation engineering from Universidad Carlos III de Madrid, Madrid, Spain, in 2012 . He is currently pursuing the Ph.D. degree with the Electronics Technology Department, Carlos III University of Madrid, Madrid.

$\mathrm{He}$ is a member of the Optoelectronics and Laser Technology Group under a FPI Researching Grant. His work focused on the acoustic detection and location of partial discharges. His current research interests include interferometric acoustic sensors and signal processing applied to acoustic signals.

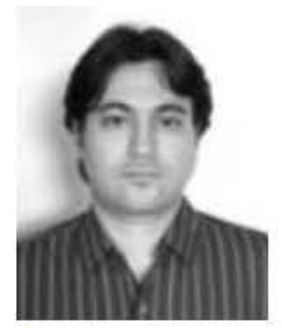

Julio E. Posada-Román was born in Barranquilla, Colombia, in 1978. He received the M.Sc. degree in advanced electronics systems from Universidad Carlos III de Madrid (UC3M), Madrid, Spain, in 2012. He is currently pursuing the Ph.D. degree at UC3M.

$\mathrm{He}$ is involved in research on fiber-optic interferometric sensors for acoustic measurements at UC3M. Since 2008, he has been a Researcher within the Optoelectronics and Laser Technology Group, Electronics Technology Department, UC3M. His current research interests include optical sensors and instrumentation for harsh environments, fiber optic interferometry, and optical sensors for industrial applications.

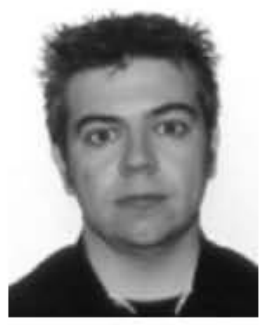

Jesús Rubio-Serrano was born in Madrid, Spain, in 1977. He received the B.Sc. degree in physics from Universidad Autónoma de Madrid, Madrid, in 2005, the M.Sc. degree in photonics technology from Universidad Autónoma de Madrid, in 2005, and the M.Sc. degree in electrical and electronics engineering from Universidad Carlos III de Madrid (UC3M), Madrid, in 2011, where he is currently pursuing the $\mathrm{Ph} . \mathrm{D}$. degree.

$\mathrm{He}$ is involved in research on the acoustic detection and location of partial discharges at UC3M. Since 2007, he has been a Doctoral Researcher with the Optoelectronics and Laser Technology Group, UC3M, under a FPI Researching Grant. His current research interests include interferometric acoustic sensors and signal processing applied to partial discharges.

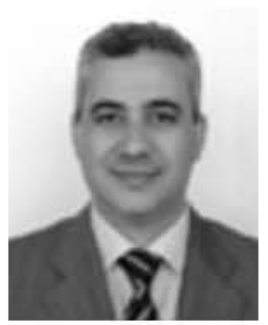

José A. Garcia-Souto (S'93-M'95) was born in Vizcaya, Spain, in 1969. He received the M.Sc. degree in telecommunication engineering from Universidad Politécnica de Madrid, Madrid, Spain, in 1995, and the Ph.D. degree from Universidad Carlos III de Madrid (UC3M), Madrid, in 2003.

He has been an Associate Professor with the Electronics Technology Department, UC3M, since 2004, and a Researcher with the Optoelectronics and Laser Technology Group, Optoelectronic Instrumentation Team, where he has been involved in the development of several instrumentation systems for power equipment monitoring. His work focused on fiber-optic sensing of acoustic emission and measurements in harsh environments. He has coordinated several research projects in these fields, particularly for high power transformers. His current research interests include optical sensors and instrumentation, fiber-optic interferometry, and optoelectronics for industrial applications. 